\title{
GUIDELINES The 2012 southern African ARV drug resistance testing guidelines
}

\author{
by the Southern African HIV Clinicians Society
}

F Conradie, D Wilson (Chairpersons of the Resistance Testing Guidelines Committee), A Basson, T de Oliveira, G Hunt, D Joel, M Papathanasopoulos, W Preiser, J Klausner, D Spencer, W Stevens, F Venter, C van Vuuren (Expert Panel Members), L Levin, G Meintjes, C Orrell, H Sunpath, T Rossouw, G van Zyl (Reviewers)

Corresponding author: F Conradie (fconradie@witshealth.co.za)

Disclaimer: Specific recommendations provided here are intended only as a guide to clinical therapy, based on expert consensus and best current evidence. Treatment decisions for patients should be made by their responsible clinicians, with due consideration for individual circumstances. The most current version of this document should always be consulted.

\footnotetext{
Following the rapid scale-up of the programme for universal access to antiretroviral therapy (ART) in southern Africa, resistance to antiretroviral medications will occur. A detectable viral load must be treated as an emergency and should trigger intensive patient tracking and adherence counselling. In contrast to the developed world, the incidence of transmitted resistance is still low in most areas in the region. Therefore, in this consensus statement we do not recommend resistance testing in HIV-infected adults upon diagnosis or ART initiation. However, baseline resistance testing is recommended for children who have been exposed to ART for prevention of mother-to-child-transmission therapy and subsequently become HIV-infected. Resistance testing is also recommended after virological failure of first- and second-line ART regimens.

S Afr J HIV Med 2012;13(4):162-167. DOI:10.7196/SAJHIVMED.874
}

\section{Opening statement}

Antiretroviral therapy (ART) has converted HIV infection from an almost universally fatal illness to a chronic manageable disease. Adherence to therapy is essential for full viral suppression and optimal immune reconstitution. If antiretroviral (ARV) drug levels are suboptimal, the risk of developing ARV drug resistance is high due to the high rate of HIV replication and the lack of proofreading capacity in the viral reverse transcriptase enzyme. Continuation of a failing ART regimen can affect both the treated individual and the community, as resistant viral strains can be transmitted to other persons.

Resistance can be minimised by uninterrupted supply of medication, scientifically sound prescribing practices, long-term adherence support, viral load (VL) monitoring, and rapid responses to demonstrated virological failure with timeous changes of therapy.

We developed consensus guidelines for HIV resistance testing that consider international best practice and the financial constraints encountered in southern Africa. The guidelines, presented here, are based on the levels of resistance in the community as reported in the 2012 World Health Organization (WHO) HIV drug resistance report. ${ }^{2}$ The North American and British resistance testing guidelines, ${ }^{3.5}$ although ideal, are not affordable nor applicable in most southern African situations. These guidelines are aimed at southern African clinicians who manage individuals with HIV infection in both the private and public sectors in our region.

Appropriate, affordable resistance testing needs to be incorporated strategically into national guidelines relevant to southern Africa. In late 2012, the South African National Department of Health sanctioned the formalisation of a National HIV Drug Resistance Working Group. All relevant stakeholders were identified and a steering committee was formed. The working group has 4 clear pillars: (i) a clinical stream; (ii) a national database development team; (iii) a laboratory team (National Health Laboratory Service (NHLS) and National Institute for Communicable Disease (NICD)); and (iv) an epidemiology stream.

The presence of a VL $>1000$ copies $/ \mathrm{ml}$ in an individual who has been receiving ART for $>6$ months constitutes an adherence emergency, and should trigger a vigorous response from the healthcare provider, including increased adherence support, before the VL measurement is repeated.

\section{Recommendations for ARV drug resistance testing 2.1 The diagnosis of HIV in children aged $<2$ years}

Genotyping at baseline to detect resistance mutations should be performed for all HIV-infected infants who have been exposed to any form of ART taken by the mother or infant for the prevention of mother-to-child transmission (PMTCT) of HIV, or who have unknown exposure to PMTCT. Infants and children are a challenging group to treat with ART, especially in resourceconstrained healthcare settings. Children and their mothers are likely to have been exposed to ARV medications in PMTCT 
programmes. Approximately 52.6\% (95\% confidence interval (CI) 37.7 67.0) of children who fail PMTCT therapy have at least one non-nucleoside reverse transcriptase inhibitor (NNRTI) mutation after single-dose nevirapine (sdNVP), and about 16.5\% (CI 8.9 - 28.3) after sdNVP combined with antepartum, postpartum or postnatal zidovudine (AZT) with or without lamivudine (3TC). ${ }^{6}$ These mutations will generally have disappeared, or may not be detectable by routine resistance testing, 2 years after the last dose of prophylactic ART. Children aged $<3$ years are treated with a boosted protease inhibitor (PI) regimen, but it is important to document NNRTI resistance, as this will have implications in the choice of second-line regimens. ${ }^{7}$ Resistance mutations in children failing ART seem to be more common than in adults. Results from baseline resistance testing will ensure the most appropriate selection of ARV drugs. Further research on appropriate drug regimens in paediatric and adolescent populations is, critically, an unmet need.

\subsection{Failure of ARV regimens}

Resistance testing is recommended for all patients (children and adults) failing first-line NNRTI-based ARV regimens, with failure defined as two VL measurements $>1000$ RNA copies $/ \mathrm{ml}$, with adherence and other issues addressed in the interval (see section 5). The accumulation of resistance mutations can be minimised by repeating the VL measurement within 3 months. ${ }^{911}$ If the first-line regimen is fully effective, then the VL should have fallen by $1.0 \log _{10}$ copies $/ \mathrm{ml}$ within 4 weeks or be undetectable by 3 months (or $<1000$ RNA copies $/ \mathrm{ml}$ in patients whose initial VL was very high). ${ }^{4}$

Resistance tests serve two purposes: (i) a fully sensitive pattern may imply that the patient is not adhering to treatment or has completely interrupted ART; and (ii) if resistance mutations are present, then the clinician, preferably together with an expert, can decide on the most appropriate second-line regimen. In patients on a stavudine (d4T)- or AZT-containing NNRTI-regimen, or on a tenofovir (TDF)-containing regimen, the importance of excluding resistance to TDF is crucial. TDF may be required as part of the nucleoside reverse transcriptase inhibitor (NRTI) backbone in second-line ART, or for treatment of hepatitis B virus $(\mathrm{HBV}) / \mathrm{HIV}$ co-infection in combination with $3 \mathrm{TC} .^{12}$

Resistance testing is recommended for all patients (children and adults) failing a PI-based ARV regimen. Failure is defined as two VL measurements $>1000$ RNA copies $/ \mathrm{ml}$, with measurements taken 3 - 6 months apart and with adherence and other issues addressed in the interval. An absence of PI mutations during PI-based therapy strongly suggests non-adherence to treatment. ${ }^{13}$ Children on any PI regimen are at high risk for PI resistance if co-treated with rifampicin (for tuberculosis). Repeated VL measurements and resistance testing are recommended for patients failing long-term PI regimens with a concurrent decline in $\mathrm{CD} 4$ count.

\subsection{Acute infection}

Recent HIV infection in adults is rarely documented; however, viral genotyping at this time may give valuable public health insights into currently circulating strains. Resistance testing recommendations for specific acute infection scenarios are shown in Table 1.

\section{Scenarios where ARV resistance testing is not recommended} 3.1 HIV diagnosis in adults and adolescents

At the current level of transmitted resistance in the community performing resistance testing in all individuals who are diagnosed with HIV infection is not cost-effective.
For pregnant women, although we do not recommend routine resistance testing, we do recommend HIV VL testing 3 months after initiating triple ARV therapy (for $\mathrm{CD} 4$ counts $<350 \mathrm{cells} / \mathrm{mm}^{3}$ ) or at the time that pregnancy is confirmed in women already receiving ART. A VL $>1000 \mathrm{RNA}$ copies/ml at this point should be regarded as an emergency, and should lead to intensive adherence support and screening for drug interactions or other reasons for failure (section 5), to minimise fetal transmission risk. The VL measurement should be repeated after 4 weeks, and, if $>1000$ copies $/ \mathrm{ml}$, HIV resistance testing and an immediate switch to a second-line ART regimen must be performed.

\subsection{ARV initiation in adults and children aged $>2$ years}

Children aged $>2$ years who stopped taking prophylactic NVP during breastfeeding more than 2 years previously do not need a resistance test prior to ARV initiation. In such cases, resistance, if present, is very unlikely to be detected by genotyping. While super-infection with a resistant viral strain is a theoretical possibility, it is considered to be so rare that performing resistance tests would not be cost-effective.

\subsection{Treatment interruptions without documented failure}

Patients who have interrupted therapy for reasons other than proven virological failure should not have HIV genotype testing performed upon presentation for subsequent ART. ${ }^{14}$ Rather, the previous ART regimen should be re-started, and VL should be measured after 3 months. Resistance mutations generally disappear rapidly in the absence of drug pressure and a reliable resistance test result may not be obtained during treatment interruptions. If the $\mathrm{VL}$ is not suppressed after adherence intervention, a resistance test can be obtained to document resistance, and an appropriate second-line regimen can be selected. ${ }^{15}$

\section{National integration of public sector laboratories}

In the public sector in South Africa, the NHLS has five centralised facilities capable of conducting sequence-based resistance testing. Currently, only two of these facilities perform routine genotyping for patient care on a large scale (Tygerberg and Johannesburg). Laboratories focus on genotyping assays, most using in-house assays, with backup from commercial assays such as Viroseq or TruGene. National surveillance is conducted at the NICD. As the ARV programme expands and patients receive treatment for longer periods of time, the capacity for resistance testing will need to be expanded. Currently, phenotyping capabilities for resistance are available, largely for research purposes, at several academic centres. Numerous research projects are underway to develop and assess more affordable and accessible approaches to resistance testing (e.g. sequencing short regions of the reverse transcriptase gene).

The Southern Africa Treatment and Resistance Network (SATuRN) has integrated the efforts of laboratories, researchers and clinicians to monitor HIV resistance patterns and advise on the clinical management of patients failing ART. The SATuRN drug resistance database systems are freely available and include two of the best public drug resistance databases in the world: the Stanford HIV Drug Resistance database and the RegaDB Clinical Management Database. SATuRN databases are used to deliver an approach to virological failure, delivering resistance genotyping, interpretation and clinical management to remote primary healthcare clinics without elaborate computer systems or infectious diseases specialists at each clinic. 
detectable plasma VL while on ART requires immediate intervention, national monitoring of the proportion of patients with detectable plasma VLs is recommended. Thresholds for response should be determined at the public health level. For example, a facility or geographic area with $>20 \%$ detectable plasma viraemia in patients on ARV who were previously undetectable, should urgently be investigated. Analyses should be performed according to demographic and geographic characteristics, and reported quarterly to national HIV treatment programmes.

The evaluation of the effect of HIV genotype testing on the selection of ARV regimens and clinical outcomes should be supported through networks of clinical programmes. Indicators to monitor the use of HIV genotype resistance assays should include: the number of assays per specified time period; the proportion of assays performed in adults, children and pregnant women; and the prevalence and type of resistance (including class of resistance and specific mutations). As the surveillance of ARV resistance and clinical use of HIV genotyping increases, additional monitoring and evaluation activities may be required.

Most settings require increased capacity for monitoring and evaluation. Resources to sustain adequate data management and interpretation are a public health priority. The net cost of incorporating resistance testing, for surveillance as well as patient management, needs to be evaluated carefully in ARV programmes in southern Africa. ${ }^{16}$

\section{Non-adherence: Causes and interventions}

The adherence requirements of ART are onerous, necessitating adherence rates $>90 \%$ for first-line NNRTI-based regimens. The best biological marker of adherence is an undetectable VL in patients on ART. The regularity of pharmacy pick-ups is also a good marker of adherence. Other strategies, including pill counts, have limited practical utility in busy clinics, and are often inaccurate. ${ }^{17}$ Pill boxes and treatment supporters may be useful in selected individuals. ${ }^{18}$

A detectable VL in patients on ART should be treated as a medical emergency, with immediate intervention, prompt evaluation by an experienced clinician and appropriate support staff (e.g. social workers, psychologists, and counsellors), and frequent follow-up. In the case of firstline virological failure, up to $50 \%$ of patients can re-suppress their $\mathrm{VL}, 7.8$ if virological failure is identified timeously, and if adherence can be improved.

In patients failing second-line therapies, and where expensive third-line options are being evaluated, newer measures such as the use of electronic pill boxes (e.g. Medication Event Monitoring System (MEMS) caps) and hair PI levels may be used, if available.

In a significant minority of cases, patients will have no resistance on resistance testing. Data from South Africa reveal that this can be as high as $15-20 \%$ where patients have been genotyped..$^{19,20}$ This means the patient is missing a large number of doses, consequently resulting in insufficient drug pressure to induce or select out existing resistance. These patients have a poorer prognosis, paradoxically, than patients with established resistance, ${ }^{21,22}$ as their poor adherence is often difficult to remedy, and may persist into subsequent regimen choices. Such patients often require the intervention of a psychologist or experienced counsellor.

Common causes of poor adherence (sections 5.1 - 5.12) are often complex and linked to social issues.

\subsection{Inadequate treatment literacy}

Most HIV programmes have extraordinary adherence rates when compared with other chronic diseases - largely due to efforts by clinic staff to ensure that patients understand HIV infection and ART. If a patient fails therapy, then some examination of the pre-ART counselling may be merited.

\subsection{Side-effects}

Side-effects are a very common reason for patients to default therapy. A careful history of often subtle but distressing side-effects (bad dreams, sleepiness, poor concentration, nausea, loss of appetite), in conjunction with a work history (shift work in particular) may allow for drug substitutions. Subtle signs of lipo-atrophy due to NRTIs are often not taken seriously by healthcare providers. Regular enquiry and immediate drug substitutions should form part of every healthcare worker encounter. Single drug substitutions should only be performed if the VL is undetectable.

\subsection{Depression and mental illness}

Undiagnosed or under-treated depression and other mental illnesses are often overlooked. The frequency of major depression is twice as high in HIV-infected patients as in matched HIV-negative patients. ${ }^{23}$ Patients with depression usually respond well to treatement with an antidepressant in combination with other non-pharmaceutical interventions. Patients who respond to antidepressant medication should be treated accordingly for at least 6 months.

\subsection{Poverty and food insecurity}

Both poverty and food insecurity have been related to poor adherence and an increased frequency of missed clinic visits. Patients often lose their jobs due to ill health in the period leading up to ART initiation. Patients should be encouraged to return to the job market as soon as is feasible, or to seek support. The need to seek work may cause patients to move away from the current clinic; therefore, referral must be facilitated. Access to available grants, social support and employment NGOs may provide additional support.

\subsection{Work-related issues}

Work-related issues, including shift work and an inability to attend clinic visits on weekdays, are a major cause of poor adherence. Long clinic waiting times and monthly medication pick-ups, may make holding down a job untenable, especially with an unsympathetic employer.

\subsection{Substance use}

Alcohol use may cause significant problems with adherence. In addition, other recreational drugs may cause problems in certain parts of the country. Use may fluctuate according to availability and peer pressure.

\subsection{Social problems}

Social problems that affect adherence include stigma, both external and internal, and poor social support networks. Perceived stigma is correlated with poor adherence. This may manifest in: a fear of tablets being found; an inability to solicit family or partner support; fear around visiting the clinic or pharmacy; or anxiety regarding an employer, neighbours or a community. Social support groups may assist in this regard.

\subsection{Denial}

ART initiation in ambivalent, conflicted patients is unlikely to have a successful outcome. The involvement of family members and partners may be an effective mechanism for addressing denial.

\subsection{Pill burden}

Pill burden is less of an issue with current regimens, but must be considered in patients who are failing treatment. Pill burden due to treatment for other 
conditions, such as hypertension or diabetes, should also be addressed. Dosing simplification, such as provision of fixed-dose combination regimens, where possible, should be a major part of advocacy within public programmes.

\subsection{Altered fertility intentions}

HIV-discordant or -concordant couples may spontaneously decide to cease their ART regimen with the intent to begin a family. Empathetic fertility counselling during ART initiation should prevent this from occurring.

\subsection{Conflict of opinions}

Conflict of opinions on the use of ARVs occurs frequently between healthcare providers, certain alternative health providers and churches. This is best addressed with an honest and non-judgmental conversation.

\subsection{Other}

Drug doses should be checked, especially in patients referred from the private sector or inexperienced sites. Drug interactions (e.g. rifampicin with a PI), absorption issues and primary acquisition of resistant virus may also result in failure.

\section{Laboratory objectives \\ 6.1 Recommendations and requirements}

A meaningful interpretation of genotypic resistance test results requires detailed knowledge of the patient's full ARV history, including drug regimens used, $\mathrm{VL}$ and $\mathrm{CD} 4$ test results, any previous resistance test results, and timelines.

It is desirable that national databases be built, using unique patient identifiers (e.g. ID numbers), to allow the easy retrieval of information for patients who have been cared for at different clinics and tested by different laboratories. Besides improving patient care and easing clinical workload, this approach is cost-effective, as it prevents unnecessary repeat testing.

All resistance test results (including clinical information and sequences obtained) should be entered into a central database, such as the one maintained by SATuRN, to enable research and surveillance.

\subsection{Genotypic ARV resistance testing: Practical issues}

Testing requires ethylenediaminetetra-acetic acid (EDTA) whole blood or EDTA plasma (purple-top tubes). Alternatively, where established, dried blood spots (DBSs) may be used. To ensure sample integrity, whole-blood and plasma samples must be maintained and shipped cooled $\left(4^{\circ} \mathrm{C}\right.$ - fridge temperature) and reach the laboratory within 48 hours. For longer delays, whole-blood specimens must be centrifuged and the plasma stored at $-20^{\circ} \mathrm{C}$ (frozen). Repeat freeze-thaw cycles must be avoided. DBSs can be maintained at room temperature for up to 4 weeks, and must be frozen at $-20^{\circ} \mathrm{C}$ if the delay is longer than 4 weeks.

Current commercial tests have been licensed for specimens with a $\mathrm{VL}$ value of at least $1000 \mathrm{RNA}$ copies/ml. If DBSs are used, then the minimum usable VL is $2000-5000 \mathrm{RNA}$ copies $/ \mathrm{ml}$. Nevertheless, many in-house assays can detect VLs of 500 - 1000 RNA copies/ml. The probability of harbouring resistance in the VL range of 500 - 1000 RNA copies $/ \mathrm{ml}$ is only marginally less than in the $1000-10000$ copies $/ \mathrm{ml}$ range. ${ }^{24}$ The acquisition of additional mutations is not necessarily associated with incremental increases in VL. ${ }^{25}$

Once a failing ART regimen has been discontinued, most resistant viral variants quickly become undetectable. Samples must therefore be obtained while the patient is still on the failing regimen or very shortly after discontinuation (to a maximum of 4 weeks).
Current test methods do not detect minority resistant viral variants (quasi-species present at less than approximately $20 \%$ of the total population) or archived resistance. ${ }^{26}$

Even in the best hands, the rate of failure to amplify virus is $5-10 \%$, so not all samples submitted to the laboratory will have a genotype result.

\subsection{Genotypic ARV resistance assays}

Currently available genotype tests evaluate only the viral reverse transcriptase and protease genes. Mutations in the genes encoding these enzymes underlie resistance to the NRTIs, NNRTIs and PIs.

Raltegravir (RAL), ${ }^{27,28}$ the first of the integrase strand-transfer inhibitors (ISTIs), is now registered in South Africa. Currently, no entry inhibitors - e.g. maraviroc (a CCR5 co-receptor inhibitor) or enfuvirtide (a fusion inhibitor) - have been registered. Future genotype tests will also need to incorporate these drug classes.

Current resistance testing is performed by means of polymerase chain reaction (PCR) amplification and sequencing/genotyping of the HIV-1 protease and reverse transcriptase genes, using commercial or validated in-house assays. The turnaround time of these assays is approximately 2 weeks. Current United States Food and Drug Administration (FDA)approved commercial assays, including ViroSeq and Trugene, can be performed at a cost of approximately R5 000 per assay. In-house assays are about $50 \%$ cheaper. Results can provide data on the presence or absence of resistance mutations, with resistance mutations interpretable by drug resistance algorithms, many of which are available online.

\section{Research priorities}

\subsection{Resistance assays}

The main thrust of research activities remains the need to develop rapid, affordable, accessible resistance assays, including:

- advocacy to drive down current commercial assay costs

- the evaluation of innovative new testing approaches, e.g. the use of more cost-effective strategies such as allele-specific assays (e.g. M184V) to determine adherence

- improved logistics using creative approaches such as DBS technology

- national standardisation of technology and reporting across the country

- continual review to ensure the incorporation of new drug classes into assays

- integrase assays

- tropism assays for CCR5 inhibitors

- the constant evaluation of new testing platforms, e.g. ultra-deep sequencing strategies

- the suitability of assays for relevant local HIV subtypes.

\subsection{Operational research activities \\ 7.2.1 Laboratory-based activities}

Laboratory-based activities should include:

- the upgrading and up-scaling of infrastructure, human resource skills, interpretation skills, and improved emergency reporting within and by the laboratory

- national data flow and reporting

- a monitoring and evaluation framework to evaluate the effect of the intervention

- ongoing cost-effectiveness modelling and analyses to assess costeffectiveness

- ensured support for strengthened national surveillance activities (i.e. increased numbers processed in realtime). 


\subsubsection{Clinical activities}

Clinical activities must include:

- strategies to develop a hierarchy of specialist support for interpretation, e.g. failure clinics

- resistance testing to support ongoing clinical research and guideline development

- strategising for the components of an appropriate standardised thirdline regimen.

\subsubsection{Basic research questions}

Future work should address:

- the contribution of detecting minority variants and their effect on patient outcome (including ultra-deep sequencing)

- the significance and role of PI mutations in the local population

- the development of a national reference facility that conducts phenotyping and other sophisticated assays to support and develop a strong scientific agenda for resistance testing.

\section{Closing comment}

These guidelines reflect the current status quo in terms of levels of HIV resistance in southern Africa in late 2012, and will be reviewed every few years. Implementation of the recommendations herein will require a drastic expansion of the laboratory capacity in the region.

Conflict of interest. All expert panel members and reviewers have completed and submitted conflict of interest disclosure forms. Disclosure information represents the previous 3 years (updated 13 October 2012) and pertains to relationships with pharmaceutical companies and medical aids. F Conradie has received research support from Bristol-Myers Squibb, Gilead, GlaxoSmithKline and Tibotec. She is also a consultant for Discovery Health. L Levin has received honoraria from Abbott, Aspen, Novagen, Optipharm and ViiV Healthcare for speaking engagements, and served as a consultant to Optipharm and Discovery Health. G Meintjes has received honoraria from Sanofi-Aventis for speaking engagements and is a consultant to Aid for AIDS. C Orrell received funding support from Merck Sharp \& Dohme (MSD) to attend a workshop, and received honoraria from Abbott for a speaking engagement. T Rossouw received honoraria from Abbott for a speaking engagement, and serves as a consultant to Discovery Health. D Spencer has received honoraria from Abbott, Adcock Ingram, Aspen, MSD and Janssen for speaking engagements. H Sunpath received research support from Gilead Sciences. G van Zyl has received honoraria from Abbott for speaking engagements. F Venter has received support from Adcock Ingram to attend conferences and honoraria from Johnson and Johnson, Merck and Tibotec for speaking engagements A Basson, T de Oliveira, G Hunt, D Joel, M Papathanasopoulos, W Preiser, J Klausner, W Stevens, C van Vuuren and D Wilson have no conflicts of interest to declare.

Acknowledgement. This work was supported and funded by the Southern African HIV Clinicians Society through an educational grant from Atlantic Philanthropies. The authors wish to acknowledge Lynsey Isherwood for her assistance in the development of this document.

\section{References}

1. Kantor R, Shafer RW, Follansbee S, et al. Evolution of resistance to drugs in HIV-1infected patients failing antiretroviral therapy. AIDS 2004;18(11):1503-1511.

2. World Health Organization (WHO). HIV Resistance Report, 2012. Geneva: WHO, 2012. http://www.who.int/hiv/pub/drugresistance/report2012/en/index.htm (accessed 9 October 2012)
3. Hirsch MS, Gunthard HF, Schapiro JM, et al. Antiretroviral drug resistance testing in adult HIV-1 infection: 2008 recommendations of an International AIDS SocietyUSA panel. Clin Infect Dis 2008;47(2):266-285. [http://dx.doi.org/ 10.1086/589297]

4. Williams I, Churchill D, Anderson J, et al. British HIV Association guidelines for the treatment of HIV-1-positive adults with antiretroviral therapy 2012. HIV Med 2012;13:1-85. [http://dx.doi.org/10.1111/j.1468-1293.2012.01029.x]

5. Thompson MA, Aberg JA, Hoy JF, et al. Antiretroviral treatment of adult HIV infection: 2012 recommendations of the International Antiviral Society - USA Panel. JAMA 2012;308(4):387-402. [http://dx.doi.org/ 10.1001/jama.2012.7961]

6. Arrive E, Newell ML, Ekouevi DK, et al. Prevalence of resistance to nevirapine in mother and children after single-dose exposure to prevent vertical transmission of HIV-1: A metaanalysis. Int J Epidemiol 2007;36;1009-1021. [http://dx.doi.org/ 10.1093/ije/dym104]

7. Martinson NA, Morris L, Gray G, et al. Selection and persistence of viral resistance in HIV-infected children after exposure to single-dose nevirapine. J Acquir Immune Defic Syndr 2007;44:148-153.

8. Sigaloff KC, Calis JC, Geelen SP, van Vugt M, de Wit TF. HIV-1-resistance-associated mutations after failure of first-line antiretroviral treatment among children in resource-poor regions: A systematic review. Lancet Infect Dis 2011;11(10):769-779. [http://dx.doi.org/10.1016/S1473-3099(11)70141-4]

9. Sigaloff KC, Ramatsebe T, Vlana R, et al. Accumulation of HIV drug resistance mutations in patients failing first-line antiretroviral treatment in South Africa. AIDS Res Hum Retroviruses 2012;28(2):171-175. [http://dx.doi.org/ 10.1089/aid.2011.0136]

10. Cozzi-Lepri A, Phillips AN, Martinez-Picado J, et al. Rate of accumulation of thymidine analogue mutations in patients continuing to receive virologically failing regimens containing zidovudine or stavudine: Implications for antiretroviral therapy programs in resource-limited settings. J Infect Dis 2009;200(5):687-697. [http://dx.doi.org/10.1086/604731]

11. Cozzi-Lepri A, Paredes, Phillips AN, et al. The rate of accumulation of nonnucleoside reverse transcriptase inhibitor (NNRTI) resistance in patients kept on a virologically failing regimen containing an NNRTI. HIV Med 2012;13(1):62-72. [http://dx.doi. org/10.1111/j.1468-1293.2011.00943.x]

12. Sunpath $\mathrm{H}, \mathrm{Wu} B$, Gordon M, et al. High rate of K65R for ART naïve patients with subtype C HIV infection failing a TDF-containing first-line regimen in South Africa. AIDS 2012 (in press). [http://dx.doi.org/10.1097/QAD.0b013e328356886d]

13. Wallis CL, Mellors JW, Venter WD, Sanne I, Stevens W. Protease inhibitor resistance is uncommon in HIV-1 subtype $\mathrm{C}$ infected patients on failing second-line lopinavir/ $\mathrm{r}$-containing antiretroviral therapy in South Africa. AIDS Res Treat 2011;769627.

14. Dlamini JN, Hu Z, Ledwaba J, Morris L, Maldarelli FM, Dewar RL, et al. Genotypic resistance at viral rebound among patients who received lopinavir/ritonavir-based or efavirenz-based first antiretroviral therapy in South Africa. J Acquir Immune Defic Syndr 2011;58(3):304-308. [http://dx.doi.org/10.1097/QAI.0b013e3182278c29]

15. El-Khatib Z, Delong AK, Katzenstein D, et al. Drug resistance patterns and virus re-suppression among HIV-1 subtype $\mathrm{C}$ infected patients receiving nonnucleoside reverse transcriptase inhibitors in South Africa. J AIDS Clin Res 2011;2(117):1000117. [http://dx.doi.org/10.4172/2155-6113.1000117]

16. de Oliveira T, Shafer RW, Seebregts C. Public database for HIV drug resistance in southern Africa. Nature 2010;464(7289):673.

17. Lima V, Harrigan R, Murray M, et al. Differential impact of adherence on long-term treatment response among naive HIV-infected individuals. AIDS 2008;22:23712380. [http://dx.doi.org/ 10.1097/QAD.0b013e328315cdd3]

18. Murphy RA, Sunpath H, Castilla C, et al. Second-line antiretroviral therapy: longterm outcomes in South Africa. J Acquir Immune Defic Syndr 2012 (in press). [http://dx.doi.org/10.1097/QAI.0b013e3182615ad1]

19. Rueda S, Park-Wyllie LY, Bayoumi A, et al. Patient support and education for promoting adherence to highly active antiretroviral therapy for HIV/AIDS Cochrane Database of Systematic Reviews 2006;3:CD001442. [http://dx.doi. org/10.1002/14651858.CD001442.pub2]

20. Rosen S, Long L, Sanne I, Stevens WS, Fox MP. The net cost of incorporating resistance testing into HIV/AIDS treatment in South Africa: a Markov model with primary data. J Intl AIDS Soc 2011;14:24.

21. Marconi VC, Sunpath H, Lu Z, et al. Prevalence of HIV-1 drug resistance after virologic failure of first-line antiretroviral therapy (ART) in a resource-limited setting: Initial results of the South Africa Resistance Cohort Study (SARCS). CID 2008;46:1589-1597.

22. Orrell C, Harling G, Lawn SD, et al. Conservation of first-line antiretroviral treatment regimen where therapeutic options are limited. Antivir ther 2007;12(1):83-88.

23. Nakasujja N, Skolasky RL, Musisi S, et al. Depression symptoms and cognitive function among individuals with advanced HIV infection initiating HAART in Uganda. BMC Psychiatry 2010;10:10-44. [http://dx.doi.org/10.1186/1471-244X-10-44]

24. Sunpath H, Lu Z, Chelin N, et al. Outcomes after virologic failure of first-line ART in South Africa. AIDS 2010;24(7):1007-1012.

25. Prosperi MC, Mackie N, Di Giambenedetto S, et al. Detection of drug resistance mutations at low plasma HIV-1 RNA load in a European multicentre cohort study. J Antimicrob Chemother 2011;66(8):1886-1896. [http://dx.doi.org/10.1093/jac/dkr171]

26. Nettles RE, Kieffer TL, Simmons RP, et al. Genotypic resistance in HIV-1-infected patients with persistently detectable low-level viremia while receiving highly active antiretroviral therapy. Clin Infect Dis 2004;39(7):1030-1037.

27. Steigbigel RT, Cooper DA, Kumar PN, et al. Raltegravir with optimized background therapy for resistant HIV-1 infection. N Engl J Med 2008;359(4):339-354. [http:// dx.doi.org/10.1056/NEJMoa0708975]

28. Lennox JL, DeJesus E, Lazzarin A, et al. Safety and efficacy of raltegravir-based versus efavirenz-based combination therapy in treatment-naive patients with HIV-1 infection: A multicentre, double-blind randomised controlled trial. Lancet 2009;374(9692):796-806. [http://dx.doi.org/10.1016/S0140-6736(09)60918-1] 